\title{
Per-Colorant-Channel Color Barcodes for Mobile Applications: An Interference Cancellation Framework
}

\author{
Henryk Blasinski, Student Member, IEEE, Orhan Bulan, and Gaurav Sharma, Fellow, IEEE
}

\begin{abstract}
We propose a color barcode framework for mobile phone applications by exploiting the spectral diversity afforded by the cyan (C), magenta (M), and yellow (Y) print colorant channels commonly used for color printing and the complementary red (R), green (G), and blue (B) channels, respectively, used for capturing color images. Specifically, we exploit this spectral diversity to realize a three-fold increase in the data rate by encoding independent data in the $C, M$, and $Y$ print colorant channels and decoding the data from the complementary $R$, $G$, and B channels captured via a mobile phone camera. To mitigate the effect of cross-channel interference among the printcolorant and capture color channels, we develop an algorithm for interference cancellation based on a physically-motivated mathematical model for the print and capture processes. To estimate the model parameters required for cross-channel interference cancellation, we propose two alternative methodologies: a pilot block approach that uses suitable selections of colors for the synchronization blocks and an expectation maximization approach that estimates the parameters from regions encoding the data itself. We evaluate the performance of the proposed framework using specific implementations of the framework for two of the most commonly used barcodes in mobile applications, QR and Aztec codes. Experimental results show that the proposed framework successfully overcomes the impact of the color interference, providing a low bit error rate and a high decoding rate for each of the colorant channels when used with a corresponding error correction scheme.
\end{abstract}

Index Terms-2-D barcodes, Aztec codes, color barcodes, interference cancellation, quick response $(\mathrm{QR})$ codes.

\section{INTRODUCTION}

O PTICAL machine readable encodings of digital data in image formats, commonly referred to as barcodes, have

Parts of this work have been presented as [1], [2].

Manuscript received December 11, 2011; revised October 12, 2012; accepted November 28, 2012. Date of publication December 11, 2012; date of current version February 6, 2013. The associate editor coordinating the review of this manuscript and approving it for publication was Dr. Debargha Mukherjee.

H. Blasinski was with the Department of Electrical and Computer Engineering, University of Rochester, Rochester, NY 14627-0126 USA. He is now with the Department of Electrical Engineering, Stanford University, Stanford, CA 94305 USA (e-mail: h.blasinski@stanford.edu).

O. Bulan was with the Department of Electrical and Computer Engineering, University of Rochester, Rochester, NY 14627-0126 USA. He is now with the Xerox Innovation Group, Webster, NY 14580 USA (e-mail: orhan.bulan@ieee.org).

G. Sharma is with the Department of Electrical and Computer Engineering, the Department of Biostatistics and Computational Biology, and is also with the Department of Oncology, University of Rochester, Rochester, NY 146270126 USA (e-mail: gaurav.sharma@rochester.edu).

Color versions of one or more of the figures in this paper are available online at http://ieeexplore.ieee.org.

Digital Object Identifier 10.1109/TIP.2012.2233483 long been utilized for tagging objects with identification and tracking data for automating sales and inventory tracking tasks. The barcode name is derived from the traditional onedimensional version of such encodings [3], where vertical bars are utilized to represent data, but is now used more generically to encompass almost all forms of data encoding in image formats that use either one-dimensional or two-dimensional (2-D) representations.

Two dimensional barcodes have recently become very popular in mobile applications for a couple of reasons. Firstly, the increasingly prevalent camera phones inherently capture 2-D images and can therefore be directly used with 2-D barcodes, unlike conventional laser-based 1-D barcode scanners that would require hardware modifications for capturing 2-D barcode images. Secondly, equally importantly, the 2-D codes offer high enough rates (per unit substrate area) to provide a viable mechanism for bridging between the physical world of print and the cyber world of the Internet. In particular, the most common uses of 2-D barcodes are for the purpose of facilitating access to online information about products and services advertised in print media such as magazines, posters, and billboards [4], [5]. The universal resource locator (URL) for the online information is embedded within the print as a 2-D barcode, which can be captured with a cell phone camera, and, upon decoding, allow the user to access the information without the tedium of manually entering the URL. Among the various options available for 2-D barcodes, for mobile applications, the (monochrome) quick response (QR) code [6], [7] is used most extensively in practice. The QR code standard [7] defines a flexible solution with competitive data rates, support for multiple character sets, features for rapid and robust synchronization under lighting and orientation variations, multiple data density designs, and built in variable error correction capability for handling differing application requirements [4]. Other popular 2-D barcode designs include the Aztec code which is extensively used in electronic ticketing or the Data Matrix common in industrial component labeling. Fig. 1 (a)-(c) illustrates examples of some common 2-D barcodes.

Although 2-D codes have higher capacity than their 1-D counterparts, innovations that further increase their capacity are of considerable interest because they allow for either: a) a reduction in the substrate foot-print that these codes consume in print media, b) additional information with the same substrate foot-print, c) greater robustness, or some combination thereof. 


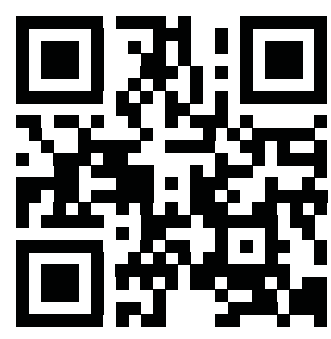

(a)

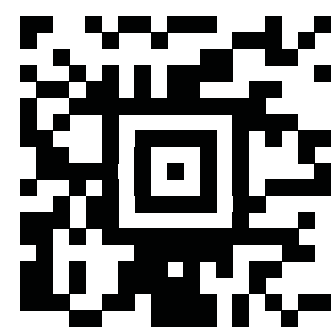

(b)

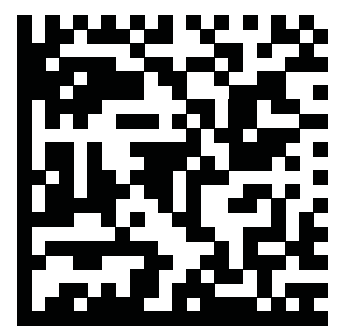

(c)

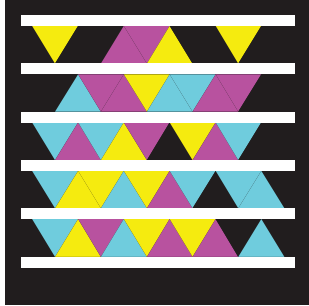

(d)

Fig. 1. Examples of common 2-D barcodes. Each barcode encodes the University of Rochester website URL: http://www.rochester.edu. (a) QR. (b) Aztec. (c) Data matrix. (d) HCCB.

Color is a common image property that is frequently leveraged to increase information density and several 2-D color barcode designs are described in the literature [8]-[14]. Sometimes, color 2-D barcodes are also referred to as 3-D codes, where color represents the third data embedding dimension. For example, Fig. 1 (d) shows a Microsoft high capacity color barcode (HCCB), a commercially used color barcode scheme. The majority of color barcodes provide increased data rates compared to 2-D monochrome barcodes by encoding data in the color of small shapes (triangles/rectangles). However, the use of colors for encoding poses challenges in the presence of inevitable tonal variations in the print and capture processes which cause changes in the colors and, to make decoding feasible, usually require part of the barcode realestate to be devoted to estimating parameters associated with the variations ${ }^{1}[15]-[17]$. Recently, [18] postulated that it is also feasible to reliably distinguish colors without a reference palette. The strategies that the proposed color barcode designs adopt for handling the print and capture variations in color also make them sensitive to lighting variations and to interseparation misregistration which can change the color of small shapes and impact the performance of the color barcodes [19]. Finally, variations across printers and cameras can also pose a challenge for these existing methods causing compromises in performance/robustness [20]-[22].

In addition to its use for increasing data rates, color is sometimes used purely for improving the visual appeal of barcodes. By leveraging extensive error correction coding it is possible to alter the typical 2-D barcode and insert graphical logos or simple, human readable graphics, while maintaining decodeability of the data embedded in the barcode. This approach, however, inherently and by design, introduces errors and severely limits the robustness of such a code to external noise sources [23].

In this paper, we propose a new framework for color barcode construction that enables extension of existing monochrome barcodes to color. Data is independently encoded in three monochrome barcodes which are then combined as the cyan (C), magenta (M), and yellow (Y) colorant channels within a single print leading to a three-fold increase in data rate compared with the corresponding monochrome barcode. The data in the $\mathrm{C}, \mathrm{M}, \mathrm{Y}$ colorant channels are decoded from the corresponding complementary red $(\mathrm{R})$, green $(\mathrm{G})$, and blue $(\mathrm{B})$ camera sensor channels. To overcome the deleterious effects of unavoidable inter-colorant interference, we use a model-based

\footnotetext{
${ }^{1}$ For these reasons, the number of colors is typically limited to fewer than 8 .
}

interference cancellation procedure that is computationally inexpensive and provides a significant performance improvement. Because the optimal parameters for the interference cancellation process are printer, camera and capture condition (e.g. lighting, perspective etc.) dependent, we propose two alternative methodologies to estimate the model parameters: a) a pilot block approach, and b) an expectation maximization (EM)-type approach. In the former, we use a customized design of the synchronization markers for the code that enables estimation of the required parameters. The latter approach on the other hand, iteratively estimates the model parameters and print-colorant channels, and hence does not require a customized design of the barcode.

Different from other color barcodes, the framework proposed in this paper encodes data as independent barcodes in $\mathrm{C}, \mathrm{M}$, and $\mathrm{Y}$ printing channels and does not encode data in the color values of individual blocks. This methodology not only enables a physical model-based interference approach for improving the decoding, but also allows re-use of a number of optimizations and improvements developed for monochrome barcodes. Thus, for example, adaptive thresholding methods that deal with variations in the illumination level and error correction coding schemes developed for correcting typical errors encountered in barcode applications are directly applicable to individual colorant channels within our proposed framework. Additionally, the proposed framework's approach of encoding independently in the colorant layers also provides better robustness to inter-colorant misregistration in printing compared with typical approaches of encoding in color, which can be very sensitive to misregistration. Experimental results demonstrate that the proposed framework is effective as well as robust to decoding errors due to cross channel interference and the resulting barcodes offer excellent performance.

This paper is organized as follows: Section II presents the proposed framework of constructing color barcodes via perchannel data encoding and the color interference cancellation algorithm. Experimental results from tests performed with the proposed barcodes are presented in Section III. Finally, Sections IV and V conclude the paper with a discussion and summary of the major findings of the paper.

\section{Color Barcodes: A Per Channel BASED FRAMEWORK}

\section{A. Data Encoding}

We propose a framework for extending monochrome barcodes to color printed using three layers of $\mathrm{C}, \mathrm{M}$, and $\mathrm{Y}$ 


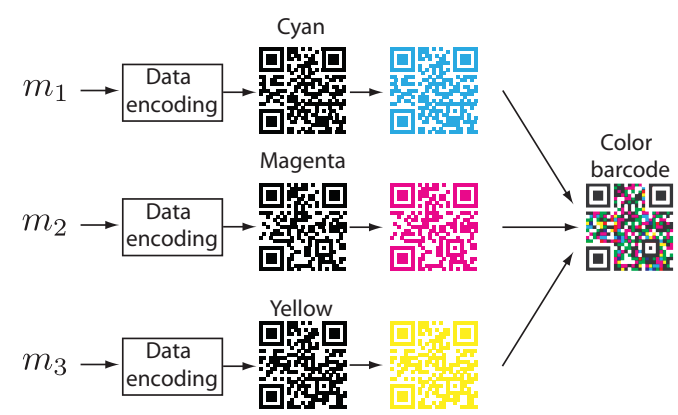

Fig. 2. Per-channel-based data encoding.

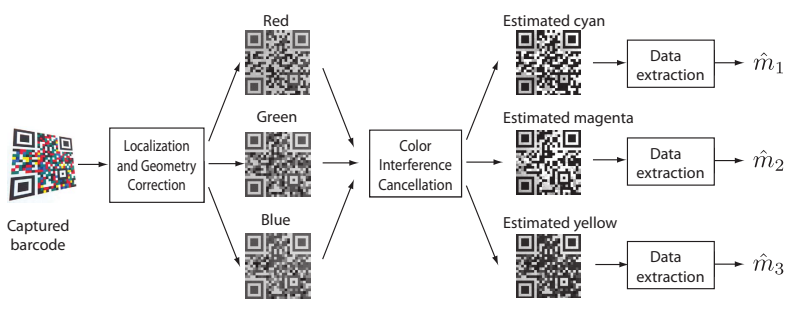

Fig. 3. Data recovery from captured barcode.

colorants corresponding to the subtractive color primaries [24]. The $\mathrm{C}, \mathrm{M}$, and $\mathrm{Y}$ colorant layers carry independent data $m_{1}, m_{2}$, and $m_{3}$, respectively, as illustrated in Fig. 2, which are printed in overlay to generate the color barcode. Data is encoded in each colorant layer utilizing the error correction coding available to monochrome counterpart to allow error recovery for data extraction from a capture of the barcode. Note that the proposed framework is applicable to any monochrome barcode and triples the encoding rate of the monochrome counterpart by utilizing $\mathrm{C}, \mathrm{M}$, and $\mathrm{Y}$ colorants available in color printing. While the encoding method is direct and a trivial extension of the monochrome methodology, the decoding of such per channel encoded color barcodes poses challeges and the primary contribution of this paper lies in methods that address these problems.

\section{B. Data Recovery}

In mobile imaging applications of interest, the decoder seeks to recover the data embedded in the barcode from an image of the barcode captured with a cell phone camera. Figure 3 shows an overview of the data recovery process. The captured barcode is first localized and separated into R, G, and B color channels, typically used in capture devices. In an ideal setting, the $\mathrm{R}, \mathrm{G}$, and $\mathrm{B}$ channels of a barcode correspond to inverted versions of the complementary $\mathrm{C}, \mathrm{M}$, and $\mathrm{Y}$ colorant layers with no cross-interactions between the camera channels and the non-complementary colorants [10]. The encoded data in C, M, and Y print colorants can then be extracted from R, G, and B capture channels, respectively.

In reality, however, there is significant cross-channel coupling among the printer and camera color channels as shown in Fig. 3. The R, G, and B camera sensor channels capture not only the complementary $\mathrm{C}, \mathrm{M}$, and $\mathrm{Y}$ printer colorant channels but also see significant interference from other noncomplementary channels. Note that the color interference is more pronounced in green and blue sensor channels due to

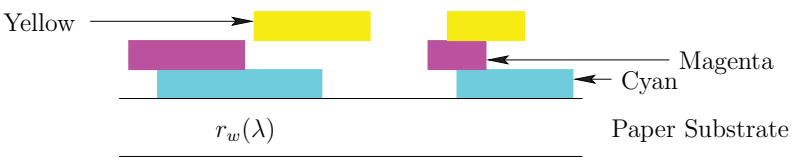

Fig. 4. Layered representation of a three $\mathrm{C}, \mathrm{M}$, and $\mathrm{Y}$ colorant print.

the spectral characteristics of the subtractive and additive color primaries [24]. In order to recover the encoded data from each color channel, we propose a color interference cancellation algorithm that estimates the $\mathrm{C}, \mathrm{M}$, and $\mathrm{Y}$ colorant layers from the R, G, and B channels of the captured barcode. The encoded data is then extracted from each estimated colorant channel. The steps shown in Fig. 3 are described next in detail.

1) Localization and Geometry Correction: In mobile applications, the printed barcode is captured in varying conditions, from different perspectives and angles. This requires to localize the barcode in the captured image. In the literature, different localization techniques exist for different barcode designs [6]-[8], [25]. For the proposed construction of color barcodes, by converting the captured barcode from color to grayscale and using the same localization and geometry correction algorithms for monochrome barcodes on the grayscale image, we simplify this process and re-use the research and engineering effort that has been invested in monochrome barcode localization techniques.

2) Color Interference Cancellation: To overcome the crosschannel color-interference, we utilize a physical model for the print and capture process. Assuming the C, M, and Y colorants are transparent (non-scattering), we model the print using the transparent version of Kubelka-Munk theory, or via the mathematically equivalent Beer-Bouguer law [26, Chap. 7]. The model can be understood by using the representation ${ }^{2}$ of Fig. 4 where the printed pattern is formed by the overlay of layers printed with $\mathrm{C}, \mathrm{M}$, and $\mathrm{Y}$ colorants on the paper substrate. The spatial pattern of a colorant layer of the barcode is represented as bi-level image $I_{i}(x, y)(i \in\{C, M, Y\})$, where $I_{i}(x, y)$ is " 1 " in regions where the $i^{t h}$ colorant is printed and "0", otherwise. Light incident on the printed color barcode is transmitted through the successive colorant layers, reflected by the paper substrate and then transmitted again through the colorant layers as it returns. Thus the spatial pattern of the spectral reflectance of the printed pattern is

$$
r(x, y ; \lambda)=r_{w}(\lambda) \prod_{i \in\{C, M, Y\}} t_{i}^{2}(x, y ; \lambda),
$$

where $(x, y)$ denote orthogonal 2-D spatial coordinates on the barcode substrate (paper), $\lambda$ denotes wavelength, $r_{w}(\lambda)$ is the spectral reflectance of the barcode substrate and

$$
t_{i}(x, y ; \lambda)= \begin{cases}t_{i}(\lambda) & I_{i}(x, y)=1 \\ 1 & I_{i}(x, y)=0\end{cases}
$$

represents the spectral transmittance of regions of a layer printed uniformly with the $i^{\text {th }}$ colorant.

\footnotetext{
${ }^{2}$ Because only on the final mathematical form of the model impacts our further development, we use a simplified description here, even though the physical model applies more generally.
} 
Denoting $d_{i}(\lambda) \stackrel{\text { def }}{=}-\log _{10}\left(t_{i}^{2}(\lambda)\right)$ as the optical density for the $i^{t h}$ colorant layer (under to and fro passage), we can re-write (1) in alternate form as

$$
r(x, y ; \lambda)=r_{w}(\lambda) 10^{-\sum_{i \in\{C, M, Y\}} d^{i}(\lambda) I_{i}(x, y)} .
$$

If the spectral sensitivity of the R, G, B capture channels is represented by $s_{k}(\lambda), k \in\{R, G, B\}$, the three channels in the image captured by the digital camera can be expressed as ${ }^{3}$ $I_{k}^{s}(x, y)=\int s_{k}(\lambda) r(x, y ; \lambda) d \lambda, k \in\{R, G, B\}$. We further approximate the spectral sensitivities of R, G, B camera color channels as Dirac delta functions $s_{k}(\lambda)=\alpha_{k} \delta\left(\lambda-\lambda_{k}\right)$ at corresponding wavelengths $\lambda_{k}, k \in\{R, G, B\}$, which allows us to write the normalized optical densities corresponding to the three captured channels as

$$
\begin{aligned}
d_{k}(x, y) & \stackrel{\text { def }}{=}-\log _{10}\left(\frac{I_{k}^{s}(x, y)}{I_{k}^{s}(W)}\right) \\
& =\sum_{i \in\{C, M, Y\}} d_{k}^{i} I_{i}(x, y)
\end{aligned}
$$

where $I_{k}^{S}(W)$ is the captured image value corresponding to the paper substrate in the $k^{t h}$ camera channel and $d_{k}^{i}=d^{i}\left(\lambda_{k}\right)$. A spatially localized estimate of $I_{k}^{s}(W)$ also makes this model tolerant to smooth variations in lighting intensity. Although the model of (4) makes several simplifying assumptions, it captures the first-order behavior of the print and capture processes. Specifically, the approximation of (3) is well-known and has also been extensively utilized for modeling color prints and photographs in other applications [28], [29]. Actual $\mathrm{R}, \mathrm{G}, \mathrm{B}$ channel responses of color capture devices, though not monochromatic, are commonly unimodal with peaks located in spectral regions complementary to the absorption peaks for $\mathrm{C}, \mathrm{M}$, and $\mathrm{Y}$ printing colorants. We note that the Dirac delta approximation for the capture sensitivities also supported by the fact that the spectra involved vary smoothly with the wavelength $\lambda$; in the limiting situation when the optical density $d_{i}(\lambda)$ does not vary with $\lambda$ over the spectral region in which the complementary capture channel sensitivity $s_{i}(\lambda)$ is nonzero, one can readily see that the Dirac-delta representation is mathematically applicable even though the physical device response is not monochromatic. The utility of the overall model of (4) has also been recently demonstrated in an alternate application [30] for the more conventional fourcolorant printing scenario.

Note that the linear relation in (4) between the densities corresponding to camera responses and print colorant channels allows us to perform interference cancellation and estimate the print colorant channels from the camera color channels provided that the optical density of the colorant layers in the camera channels $d_{k}^{i}$,s for all $k \in\{R, G, B\}$ and $i \in\{C, M, Y\}$ are available ${ }^{4}$. Specifically, denoting by $\mathbf{D}$ the matrix whose $i^{\text {th }}$ column is $\left[d_{R}^{i}, d_{G}^{i}, d_{B}^{i}\right]^{T}$, we see that under the model

\footnotetext{
${ }^{3}$ In actual practice, due to variations in lighting, the model does not apply on an absolute basis. The spatial variations in lighting, however, impact all colorant channels identically and can be handled after our proposed processing in much the same way as they are currently tackled for monochrome barcodes [15], [20], [27].

${ }^{4}$ Also note that the model is not impacted by a power-law transform, commonly employed for "gamma correction."
}

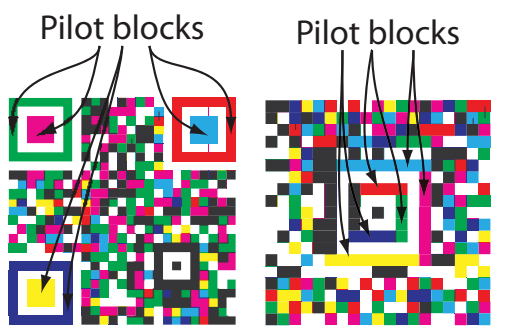

(a)

(b)

Fig. 5. Pilot blocks localization in selected barcodes. (a) QR. (b) Aztec.

of (4) the printed binary channels $\mathbf{I}=\left[I_{C}, I_{M}, I_{Y}\right]^{T}$ at a given location $(x, y)$ are obtained from the recorded density $\mathbf{d}=\left[d_{R}, d_{G}, d_{B}\right]^{T}$ at that location by

$$
\mathbf{I}=\mathbf{D}^{-1} \mathbf{d} \text {. }
$$

Based on (5), our goal is to estimate the printed binary channels I from the density matrix $\mathbf{d}$ calculated from the captured barcode. In a practical application, however, $\mathbf{D}$ is typically unknown, which varies depending on several factors including printer, capture device, illumination etc. We next propose two alternative approaches for estimating $\mathbf{D}$.

a) Pilot Block Approach: In our first method for estimating $\mathbf{D}$, we propose a customized design of color barcodes, where we include six pilot blocks in the design of the barcode. These pilot blocks correspond to six combinations of the colorant layers, in particular C, M, Y, CM, CY, and MY, where the latter three represent the printer blue, green, and red, respectively ${ }^{5}$.

For encoding pilot blocks, we use the positioning marks available in several monochrome barcodes to allow quick detection of the barcode pattern by the decoder. These positioning marks do not carry data and enable synchronization at the decoder. Figure 5 shows example pilot block encoding in positioning marks for both QR and Aztec codes. For obtaining an estimate of $\mathbf{D}$, we use the R, G, B values sensed by the digital camera for the positioning marks shown in Fig. 5 that correspond to printing of the colorant combinations $\mathrm{C}, \mathrm{M}$, $\mathrm{Y}, \mathrm{CM}, \mathrm{CY}$, and MY. After finding the positioning marks in the barcode the normalized R, G, B channel optical densities are computed for each pixel contained inside the positioning marks. An estimate of $\mathbf{D}$ is then calculated from these observed values and the known input values $I_{C}, I_{M}$, and $I_{Y}$ for these pixels via a constrained least-squares fit to the model of (5), where $\mathbf{D}$ is restricted to be positive.

b) EM-Type Approach: We also propose a general approach for estimation of $\mathbf{D}$ without requiring pilot blocks. For this purpose, we use an iterative algorithm to estimate I and $\mathbf{D}$ simultaneously, treating these quantities as hidden and latent variables in an expectation maximization (EM) style formulation. Specifically, we relax the integer constraints on I, treating these instead as variables constrained to lie between 0 and 1 , which may alternatively be interpreted as probabilities of the corresponding colorant being printed at the given pixel location. We then formulate the problem of

\footnotetext{
${ }^{5}$ In terminology used in the context of modeling color printers, these are a subset of the so-called Neugebauer printing primaries [24].
} 


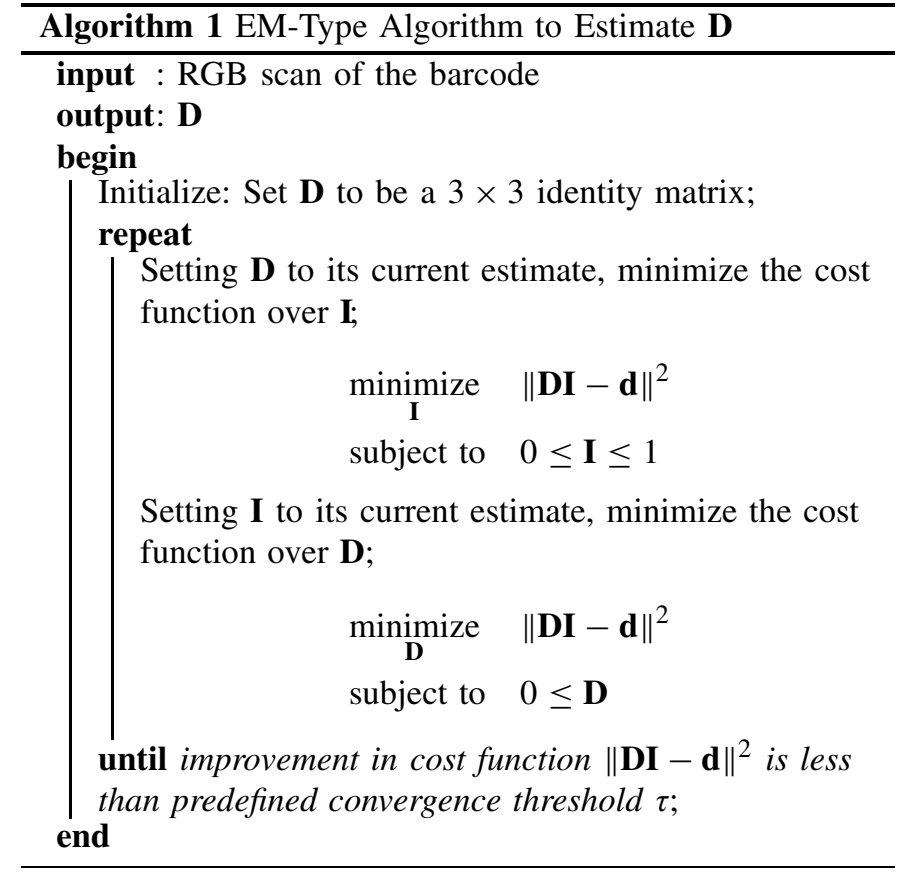

estimating $\mathbf{I}$ and $\mathbf{D}$ as an optimization problem that seeks to minimize the cost function $\|\mathbf{D I}-\mathbf{d}\| \|^{2}$. Because we have hidden and latent variables in the cost function, we formulate the overall estimation process as an alternating least squares minimization as indicated in Algorithm 1. Each iteration of the algorithm alternates over minimization of the cost function over $\mathbf{D}$ and $\mathbf{I}$ in two sequential steps. In this process, the constraint $0 \leq \mathbf{I} \leq 1$ is imposed based on our proposed relaxation and additionally, based on the physical model, we constrain $\mathbf{D}$ to include only positive entries. The algorithm is initialized by setting $\mathbf{D}$ to a $3 \times 3$ identity matrix ${ }^{6}$. The algorithm is terminated once the improvement in the objective function falls below a predetermined threshold $\tau$. Note that the relaxation of the integer constraint on $\mathbf{I}$ in Algorithm 1 also renders the problem computationally tractable; the integer constrained problem is NP hard [31]. For computational and memory efficiency, the computations for Algorithm 1 may be performed over a subset of the pixels in the image. The print colorant channels I can then be estimated by using (5) with the estimate of $\mathbf{D}$ obtained via Algorithm 1 .

Note that convergence of the alternating minimization algorithm is assured because each step in the iteration reduces the value of the cost function. In view of the probabilistic interpretation of the relaxation we introduced, the proposed methodology to find $\mathbf{D}$ is an EM-type algorithm that finds the local optima in the vicinity of the initialization point [32, Chap. 9].

3) Data Recovery from Color Channels: Performing the color interference cancellation we obtain three grayscale images corresponding to $\mathrm{C}, \mathrm{M}$, and $\mathrm{Y}$ printing layers. Data can then be recovered from each of these grayscale

\footnotetext{
${ }^{6}$ This corresponds to the idealized assumption that the RGB sensor channels capture the printing in the complementary CMY colorants with no color interference.
}

images by using a data recovery algorithm for the corresponding monochrome barcode. By design, our framework is capable of exploiting most existing methods for monochrome barcode encoding and decoding. Next, we provide a brief summary of the data recovery process emphasizing aspects that are new to the framework. For concreteness, we model our description after the decoder structure typically used for QR and Aztec barcodes, the most common monochrome 2-D barcodes for mobile applications ${ }^{7}$. The data recovery process consists of binarizing the grayscale image to reduce the impact of print and capture noise, followed by data decoding.

a) Binarization: In order to binarize the separated color channels, we utilize three different thresholding algorithms including the local thresholding algorithm commonly used in several monochrome barcodes [6], [7], [33], [34].

Fixed Thresholding: The simplest thresholding algorithm for binarization is using a fixed threshold, one value per color channel. Thresholds for the color channels can be selected differently depending on the interference level seen in each channel. Though simple, the limitation of this algorithm is that once a threshold is fixed, it does not adapt to changes in printing colorants, capture device spectral sensitivities and lighting variations.

Adaptive Thresholding: When the pilot block approach is used for color interference cancellation, six combinations of the colorant layers, C, M, Y, CM, CY, and MY, are printed in positioning marks as shown in Fig. 5. In the adaptive thresholding algorithm, we utilize the intensity values at the positioning marks to set a threshold value for a color channel. In particular, we set the thresholds for the estimated $\mathrm{C}, \mathrm{M}$, and $\mathrm{Y}$ channels as:

$$
\begin{aligned}
t_{C} & =\frac{\max \left(I_{R}^{C}, I_{R}^{C M}, I_{R}^{C Y}\right)+\min \left(I_{R}^{M}, I_{R}^{Y}, I_{R}^{M Y}\right)}{2} \\
t_{M} & =\frac{\max \left(I_{G}^{M}, I_{G}^{C M}, I_{G}^{M Y}\right)+\min \left(I_{G}^{C}, I_{G}^{Y}, I_{G}^{C Y}\right)}{2} \\
t_{Y} & =\frac{\max \left(I_{B}^{Y}, I_{G}^{C Y}, I_{G}^{M Y}\right)+\min \left(I_{G}^{C}, I_{G}^{M}, I_{G}^{C M}\right)}{2}
\end{aligned}
$$

where $I_{i}^{j}$ is the intensity in the $i^{\text {th }}$ color channel $i \in\{R, G, B\}$ of the $j^{\text {th }}$ pilot block $j \in\{C, M, Y, C M, C Y, M Y\}$ and $t_{C}$, $t_{M}$, and $t_{Y}$ are the threshold values for the estimated $\mathrm{C}, \mathrm{M}$, and $\mathrm{Y}$ channels. Adaptive thresholding algorithm can only be used when the barcode is designed in a customized fashion as described in Sec. II-B.2.a.

Local Thresholding: The local thresholding algorithm is extensively used in monochrome barcodes to account for illumination variations across the captured image. In this thresholding algorithm, the image is first divided into nonoverlapping blocks and average intensity is calculated for each block. The threshold for a given block is then determined using the intensity of the blocks in a $5 \times 5$ neighborhood of the given block. The threshold across the barcode substrate varies because a different threshold is assigned for each block by using intensities of the local neighborhood only.

\footnotetext{
${ }^{7}$ Subsequently, in Appendix, we also highlight an application of the proposed framework to an entirely different type of high capacity barcode.
} 

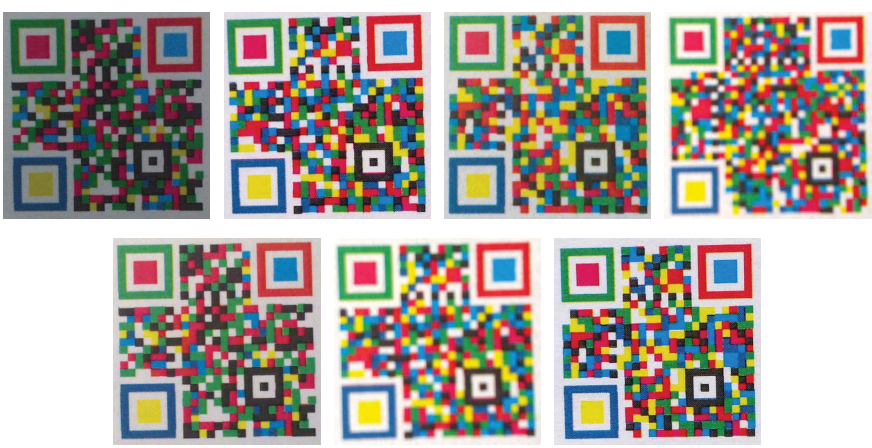

Fig. 6. Sample color QR code images.

b) Data Decoding: Using the binarized C, M, and Y channel images, first bit values corresponding to each of the barcode blocks are obtained, which are then utilized by the error correction decoder to recover the embedded message. Both the QR and the Aztec codes, utilize Reed-Solomon (RS) codes for error correction coding, which work well within the computational constraints of mobile devices.

\section{EXPERIMENTAL RESULTS}

\section{A. Experimental Set-Up and Performance Metrics}

In order to evaluate the performance of the proposed framework, we select two popular monochrome 2-D barcodes and extend their functionality to color. We choose the QR and Aztec codes because they are most extensively used in mobile applications.

We evaluate the performance of the proposed construction of color barcodes by using three different metrics: bit error rate (BER), synchronization success rate (SSR) and decoding success rate (DSR). The bit error rate (BER) is the percentage of bits in error in the extracted bit stream compared to the originally embedded bit stream prior to application of error correction mechanisms. When compared across different algorithms applied to the same barcode captures, it provides a performance measure for evaluating the effectiveness of the proposed color interference cancellation framework. The second metric, synchronization success rate (SSR), gives the percentage of color barcodes, for which the decoder can successfully locate the barcode in the image. We compare the synchronization rate of the proposed color barcodes with that of monochrome barcodes to evaluate whether the color barcode construction degrades synchronization performance. Finally, the decoding success rate (DSR) is the percentage of the synchronized barcodes, where the encoded data is recovered without any errors after error correction coding. The decoding success rate (DSR) serves as a measure of the overall performance of the proposed color interference cancellation methodology in estimating the data embedded independently in print colorant channels.

We generated per-colorant-channel color versions of $\mathrm{QR}$ and Aztec codes in our proposed framework by using the $\mathrm{Zint}^{8}$ open source encoder to generate encodings of independent data (URLs) in each of the C, M, and Y colorant channels, which

\footnotetext{
${ }^{8} \mathrm{http} / / /$ sourceforge.net/projects/zint/
}
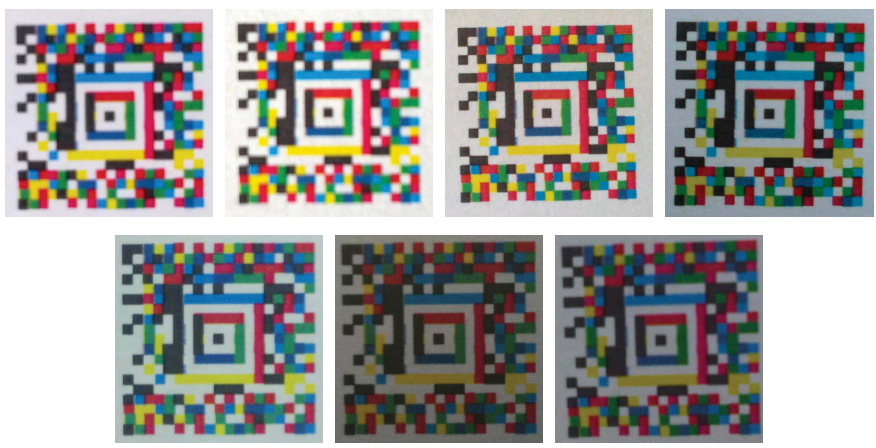

Fig. 7. Sample color Aztec code images.

were then combined to produce the final color versions of the barcode for printing. The color barcodes, thus constructed, were printed on several different office printers and then captured with a variety of mobile phones, as listed in Table I, under typical lighting conditions (indoors, outdoors, fluorescent, and incandescent). The highest resolution and the lowest JPEG compression rate are selected for the phones that allow control of image acquisition settings. We capture images in focus from an approximate distance of $15 \mathrm{~cm}$ from the printed barcode. Figures 6 and 7 show example images of barcodes captured with several different mobile phone cameras.

Acquired barcodes are decoded using five different methods. The first two methods are implemented for benchmarking and extract the data by performing a thresholding operation on the $\mathrm{R}, \mathrm{G}$, and $\mathrm{B}$ channels of the acquired color barcode without interference cancellation. Depending on the algorithm, these methods are called localized $(L T)$ and adaptive $(A T)$ thresholding. The remaining three methods implement the proposed interference cancellation scheme as described in Section II-B.2 to obtain improved estimates of C, M, and Y colorant channels as described in Sec. II-B.3.a, using either the EM-type approach $(E M)$ or the pilot block approach, which is combined with localized $(P B+L T)$ or adaptive $(P B+A T)$ thresholding algorithms. For the EM-type approach, a value of $\tau=10^{-5}$ was used for the convergence check. Both, for our proposed interference cancellation framework, and for alternative approaches used for benchmarking, a Java based implementation was used for the data recovery, which made use of the core functionality for monochrome QR and Aztec barcode decoding from the open source ZXing ${ }^{9}$ decoder and the EMJL ${ }^{10}$ library for matrix operations.

Because the overall performance of 2-D barcodes also depends on the size of the features used in the barcode and the level of error correction coding redundancy ${ }^{11}$, we conducted our experiments in two phases. In the first phase, we evaluate the performance in terms of bit error rate prior to error correction decoding, considering several different sizes for the barcode features and several different printers. Based on the results from this first phase, we then select a suitable barcode feature size, which we utilize in the second phase

\footnotetext{
${ }^{9}$ http://code.google.com/p/zxing/

${ }^{10} \mathrm{http} / / /$ code.google.com/p/efficient-java-matrix-library/

${ }^{11}$ Specifically, the QR code allows different choices for the level of redundancy for the error correction coding.
} 
of our experiments where we also consider different levels of error correction coding redundancy and report performance in terms of the three metrics previously indicated. Furthermore, because the results of the first experiment demonstrate small variability across printers, in the second phase, we narrow our attention to one printer. This approach allows us to explore the available design space without facing the explosion of combinatorial possibilities for all parameters.

\section{B. Phase I Experiments}

In the first phase of experiments, we evaluate the impact of the feature size and printer variability on the performance of the proposed color barcodes in order to select parameters (e.g. feature size, printer) for the second phase. We generate the percolorant-channel QR and Aztec color barcodes in four different sizes: 15, 20, 25 and $30 \mathrm{~mm}$ by using the same barcode but different feature sizes, i.e. the dimensions of the smallest blocks that encode each bit of data in the data encoding regions. The barcodes were printed using the ten different laser and inkjet printers listed in Table I. Each printed barcode was captured five times using ten different phones. This resulted in a database containing $2000 \mathrm{QR}$ and 2000 Aztec code images.

The acquired barcodes in the database are decoded using the four previously defined methods denoted as: $L T, A T$, $P B+L T$, and $P B+A T$, where first two methods, included for benchmarking purposes, operate by simply thresholding in individual R, G, B channels. Tables II and III list the resulting bit error and the synchronization success rates for color QR and Aztec codes, where BER and SSR denote the bit error rate, and synchronization success rate, respectively. As shown in the tables, the interference cancellation based techniques produce consistently lower bit error rates for all channels. In the case of cyan and magenta, the best performance is obtained when interference cancellation is combined with local thresholding $(P B+L T)$ accounting for lighting variation across the barcode. For the yellow channel, the adaptive thresholding produces the best results because it takes into account prior knowledge of color intensities obtained from pilot blocks. Also from the data presented in the tables it is clear that the synchronization rate changes as the feature size gets smaller. For both QR and Aztec codes, the synchronization rate drops steeply as the barcode size reduces from $20 \mathrm{~mm}$ to $15 \mathrm{~mm}$, which implies that the feature size corresponding to our $15 \mathrm{~mm}$ barcode size is not sufficient for reliable barcode detection. This observation agrees with the earlier work in color barcode synchronization, [35] reports that features below $0.75 \mathrm{~mm}$ demonstrate consistently higher error rates. We have also noted that average SSR scores obtained for 20, 25 and $30 \mathrm{~mm}$ barcodes are consistent with equivalent measures presented in [36], [37]. Finally, these results agree with our observation that the typical feature size for commercially used monochrome barcodes is $0.75 \mathrm{~mm}$, which corresponds to the $20 \mathrm{~mm}$ barcode used in our experiment. Based on the results in the first phase of our experiments and in the aforementioned sources, we used the feature size corresponding to the $20 \mathrm{~mm}$ barcode in the second phase of our experiments.
TABLE I

DeVices Used in Experiments. ${ }^{\dagger}$ AND ${ }^{+}$Indicate Devices Used ONLY IN FIRST AND SECOND ROUND EXPERIMENTS, RESPECTIVELY

(a) Phones

\begin{tabular}{|l|l|c|c|}
\hline ID & Make and Model & Sensor Size, MP & Autofocus \\
\hline \hline 1 & LG Ally & $\dagger$ & $\checkmark$ \\
2 & iPhone 4 $^{\dagger}$ & 3.2 & $\checkmark$ \\
3 & iPhone 3GS & 3.0 & $\checkmark$ \\
4 & iPhone 3G $^{\dagger}$ & 2.0 & \\
5 & Nokia C6 & 5.0 & $\checkmark$ \\
6 & Samsung Intercept & 3.2 & $\checkmark$ \\
7 & Motorola Droid X & 8.0 & $\checkmark$ \\
8 & Motorola Droid & 5.0 & $\checkmark$ \\
9 & Blackberry 8900 Curve & 3.2 & $\checkmark$ \\
10 & Nokia N900 & 5.0 & $\checkmark$ \\
11 & LG VX9900 + & 2.0 & $\checkmark$ \\
\hline
\end{tabular}

(b) Printers. $\ddagger$ indicates a solid ink printer.

\begin{tabular}{|c|l|c|c|}
\hline ID & Make and Model & Laser & Inkjet \\
\hline \hline 1 & HP OfficeJet 6500 & & $\checkmark$ \\
2 & HP Color LaserJet 4700 & $\checkmark$ & \\
3 & Ricoh Aficio MPC2550 & $\checkmark$ & \\
4 & HP Color LaserJet 4650 & $\checkmark$ & \\
5 & Epson Stylus NX215 & & $\checkmark$ \\
6 & Dell Printer 948 & & $\checkmark$ \\
7 & Xerox WorkCentre 7665 & $\checkmark$ & \\
8 & Xerox Phaser 8860 & & $\checkmark$ \\
9 & Ricoh Aficio MPC4501 & $\checkmark$ & \\
10 & HP Color LaserJet 5550 & $\checkmark$ & \\
\hline
\end{tabular}

Using the same set of barcodes, we also evaluate the performance across the printers shown in Table I. Figure 8 presents the bit error rate distributions for each printer. In the figure, the red line shows the median, and the top and bottom of the box denote the $25^{t h}$ and $75^{\text {th }}$ percentiles of the bit error rates, respectively. Whiskers represent the extreme values which are not outliers denoted by a "+". Note that the yellow channel has the highest variability and the highest error rates across the printers because in many commercial printers the yellow channel contains a random dot pattern that is used for identifying the printer for anticounterfeiting purposes [38], which interferes with the data encoded in yellow channel. The prints from Printer 4 exhibited black bands in the print caused by imperfections on the photoreceptor due to which the image quality was rather poor, which, in turn, resulted in the high error rates. In all other cases, the effect of printer variation on the bit error rate is small. Therefore, we selected a single printer for the second part of our experiments to allow us to explore alternatives for the redundancy used in the error correction coding.

\section{Phase II Experiments}

In this experiment we evaluate the performance of the proposed framework in terms of the three performance metrics (BER, DSR, SSR) defined in Sec. III-A. Because the decoding success rate (DSR) also depends on the redundancy used in the error correction coding, we used QR codes with the four possible error correction level settings specified in the 
TABLE II

Percent BERs and SSRs (Synchronization Success Rates) For Color QR Codes With the Proposed Interference Cancellation (Int. Cancellation) Approaches (PB+AT, PB+LT) And Alternative Approaches (AT, LT)

\begin{tabular}{|c|c|c|c|c|c|c|c|c|c|c|c|c|c|}
\hline \multirow{3}{*}{ Size } & & \multicolumn{4}{|c|}{ Estimated Cyan } & \multicolumn{4}{|c|}{ Estimated Magenta } & \multicolumn{4}{|c|}{ Estimated Yellow } \\
\hline & & \multicolumn{2}{|c|}{ Int. cancellation } & \multirow{2}{*}{ AT } & \multirow{2}{*}{ LT } & \multicolumn{2}{|c|}{ Int. cancellation } & \multirow{2}{*}{ AT } & \multirow{2}{*}{ LT } & \multicolumn{2}{|c|}{ Int. cancellation } & \multirow{2}{*}{ AT } & \multirow{2}{*}{ LT } \\
\hline & & $\mathrm{PB}+\mathrm{AT}$ & $\mathrm{PB}+\mathrm{LT}$ & & & $\mathrm{PB}+\mathrm{AT}$ & $\mathrm{PB}+\mathrm{LT}$ & & & $\mathrm{PB}+\mathrm{AT}$ & $\mathrm{PB}+\mathrm{LT}$ & & \\
\hline \multirow{2}{*}{$15 \mathrm{~mm}$} & BER & 9.23 & 1.72 & 5.09 & 1.49 & 8.37 & 5.69 & 12.28 & 8.71 & 6.88 & 6.48 & 20.66 & 17.45 \\
\hline & SSR & 82.36 & 82.36 & 82.16 & 82.16 & 82.36 & 82.36 & 80.36 & 82.16 & 82.36 & 82.36 & 82.16 & 82.16 \\
\hline \multirow{2}{*}{$20 \mathrm{~mm}$} & BER & 5.02 & 0.87 & 2.49 & 2.16 & 5.57 & 4.25 & 10.44 & 7.61 & 5.13 & 5.39 & 18.18 & 17.59 \\
\hline & SSR & 92.40 & 92.60 & 92.60 & 92.60 & 92.60 & 92.60 & 92.00 & 92.00 & 92.60 & 92.60 & 92.60 & 92.60 \\
\hline \multirow{2}{*}{$25 \mathrm{~mm}$} & BER & 1.21 & 0.55 & 0.57 & 0.47 & 3.11 & 3.03 & 8.77 & 5.76 & 3.42 & 4.56 & 15.14 & 15.31 \\
\hline & SSR & 91.40 & 91.40 & 91.20 & 91.40 & 91.40 & 91.40 & 90.80 & 91.20 & 91.40 & 91.40 & 91.20 & 91.40 \\
\hline \multirow{2}{*}{$30 \mathrm{~mm}$} & BER & 0.70 & 0.58 & 0.15 & 0.27 & 2.73 & 2.47 & 7.31 & 4.60 & 3.37 & 3.88 & 16.85 & 13.64 \\
\hline & SSR & 90.16 & 90.16 & 90.16 & 90.16 & 90.16 & 90.16 & 90.16 & 89.96 & 90.16 & 90.16 & 89.96 & 90.16 \\
\hline \multirow{2}{*}{ Total } & BER & 3.92 & 0.91 & 2.00 & 1.09 & 4.87 & 3.82 & 9.63 & 6.63 & 4.65 & 5.05 & 17.64 & 15.97 \\
\hline & SSR & 89.08 & 89.13 & 89.03 & 89.08 & 89.13 & 89.13 & 88.33 & 88.83 & 89.13 & 89.13 & 88.98 & 89.08 \\
\hline
\end{tabular}

TABLE III

PERCEnt BERs and SSRs (Synchronization Success Rates) Rates for Color AzTeC Codes With the Proposed Interference CAncellation (Int. Cancellation) Approaches (PB+AT, PB+LT) And Alternative Approaches (AT, LT)

\begin{tabular}{|c|c|c|c|c|c|c|c|c|c|c|c|c|c|}
\hline \multirow{3}{*}{\multicolumn{2}{|c|}{ Size }} & \multicolumn{4}{|c|}{ Estimated Cyan } & \multicolumn{4}{|c|}{ Estimated Magenta } & \multicolumn{4}{|c|}{ Estimated Yellow } \\
\hline & & \multicolumn{2}{|c|}{ Int. cancellation } & \multirow{2}{*}{ AT } & \multirow{2}{*}{ LT } & \multicolumn{2}{|c|}{ Int. cancellation } & \multirow{2}{*}{ AT } & \multirow{2}{*}{ LT } & \multicolumn{2}{|c|}{ Int. cancellation } & \multirow{2}{*}{ AT } & \multirow{2}{*}{ LT } \\
\hline & & $\mathrm{PB}+\mathrm{AT}$ & $\mathrm{PB}+\mathrm{LT}$ & & & $\mathrm{PB}+\mathrm{AT}$ & $\mathrm{PB}+\mathrm{LT}$ & & & $\mathrm{PB}+\mathrm{AT}$ & $\mathrm{PB}+\mathrm{LT}$ & & \\
\hline \multirow{2}{*}{$15 \mathrm{~mm}$} & BER & 2.01 & 2.16 & 1.94 & 2.18 & 1.17 & 1.32 & 1.45 & 1.51 & 1.70 & 2.25 & 2.08 & - \\
\hline & SSR & 20.61 & 15.56 & 21.21 & 16.16 & 10.51 & 10.10 & 10.91 & 1.41 & 7.47 & 1.01 & 0.40 & 0.00 \\
\hline \multirow{2}{*}{$20 \mathrm{~mm}$} & BER & 1.24 & 1.51 & 1.22 & 1.39 & 0.86 & 1.00 & 1.00 & 1.34 & 1.35 & 1.68 & 1.23 & - \\
\hline & SSR & 48.79 & 40.69 & 54.86 & 46.15 & 41.30 & 30.57 & 35.22 & 8.70 & 29.55 & 15.18 & 4.45 & 0.00 \\
\hline \multirow{2}{*}{$25 \mathrm{~mm}$} & BER & 0.85 & 1.14 & 0.82 & 1.07 & 0.79 & 0.97 & 0.90 & 1.23 & 0.98 & 1.36 & 1.47 & 2.38 \\
\hline & SSR & 69.29 & 63.64 & 73.33 & 69.90 & 64.24 & 49.29 & 54.55 & 20.40 & 49.09 & 33.74 & 9.90 & 1.41 \\
\hline \multirow{2}{*}{$30 \mathrm{~mm}$} & BER & 0.68 & 0.86 & 0.59 & 0.76 & 0.73 & 0.80 & 0.89 & 1.19 & 1.01 & 1.22 & 1.61 & 1.72 \\
\hline & SSR & 75.15 & 70.91 & 80.81 & 77.17 & 74.75 & 54.95 & 60.40 & 23.84 & 56.77 & 38.99 & 15.15 & 1.62 \\
\hline \multirow{2}{*}{ Total } & BER & 0.99 & 1.20 & 0.94 & 1.11 & 0.81 & 0.94 & 0.95 & 1.24 & 1.11 & 1.36 & 1.52 & 2.03 \\
\hline & SSR & 53.46 & 47.70 & 57.55 & 52.35 & 47.70 & 36.23 & 40.27 & 13.59 & 35.73 & 22.23 & 7.48 & 0.76 \\
\hline
\end{tabular}

standard [7]: $L$ (lowest), $M, Q$ and $H$ (highest). Based on our observations from the first phase of our experiments, we set the feature size to the smallest value that offers reasonable performance in terms of bit error and synchronization rates, i.e. $0.75 \mathrm{~mm}$. With the feature size and data payload fixed, the sizes of the barcodes then vary depending on the error correction level.

Corresponding to each colorant channel of a color barcode, we also generate a monochrome black and white 2-D barcode counterpart for benchmarking with the same encoded data, and having the same level of error correction and feature size as the color version. The generated barcodes were printed on HP Color LaserJet $4700 \mathrm{dn}^{12}$ and the printed barcodes are captured with nine ${ }^{13}$ different mobile phones from the set shown in Table I(a). This produced a database containing 180 images of color and 540 images of monochrome QR codes, 45 images of color and 135 images of monochrome Aztec codes.

The captured color barcodes are decoded using five different decoding methodologies as described in the experimental setup (Sec. III-A) and the monochrome barcodes are decoded

\footnotetext{
${ }^{12}$ Note that we printed the barcodes on a single printer as the performance does not vary significantly across the printers as shown in Sec. III-B.

${ }^{13}$ The phones used in phase one were not all available for the second phase experiments.
}

using the conventional ZXing decoder. The decoding and synchronization rates for $\mathrm{QR}$ codes with different error correction levels are shown in Table IV. The overall synchronization rate for color QR codes is quite high (95\%) and comparable (or higher) than the synchronization rate obtained for monochrome QR code $(90 \%)$. In all cases, the decoding rate in the cyan channel offers the highest performance (approx. 90\%) irrespective of the decoding method used. For the magenta channel, the best decoding rate is obtained with the pilot block interference cancellation algorithm followed by adaptive thresholding $(P B+A T)$, and for the yellow channel EM-type approach for color interference cancellation achieves the best performance. Note that the decoding success for the yellow channel without interference cancellation is less than 3\% irrespective of the thresholding algorithm applied. The proposed interference cancellation algorithms significantly improve the decoding performance in the yellow channel and make it feasible to encode data in this channel, allowing higher data rates for the 2-D barcodes used in mobile applications. The effect of interference cancellation is also shown in Fig. 9 for a QR code, where the separated color channels with and without color interference cancellation are shown sideby-side, illustrating the benefit of the proposed interference cancellation algorithm. 


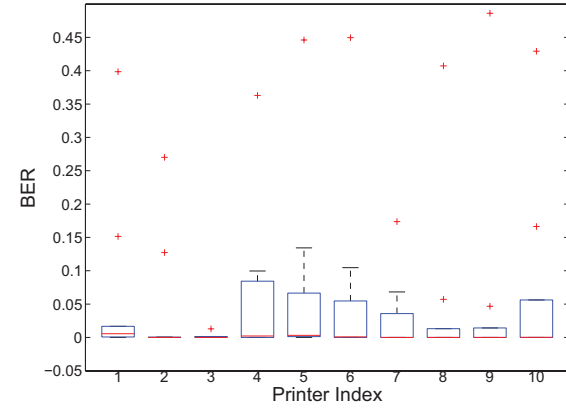

(a)

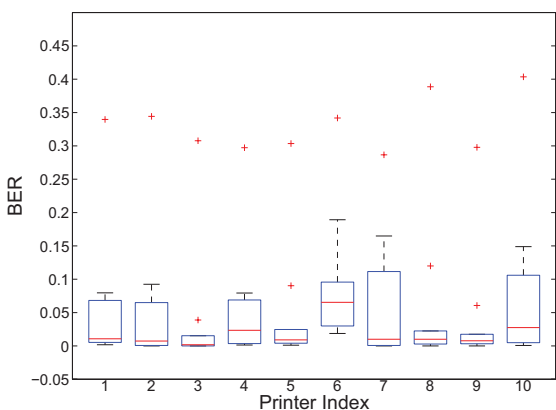

(d)

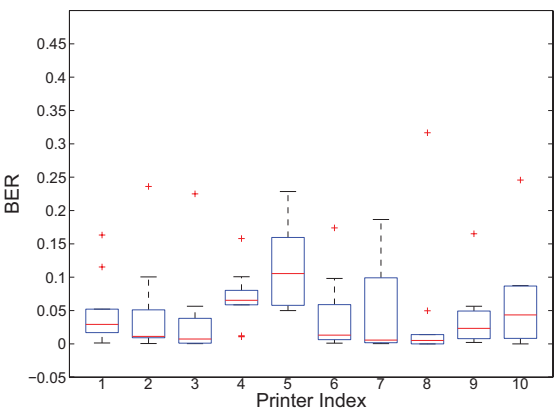

(g)

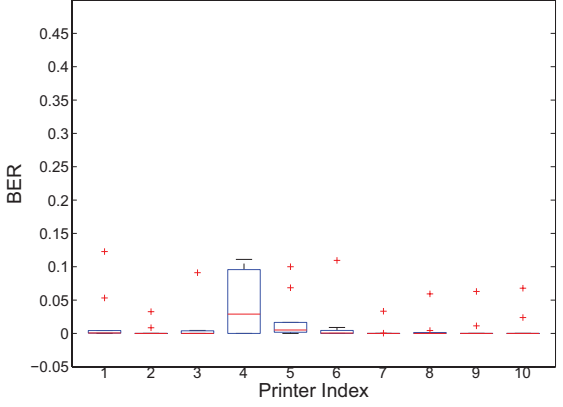

(b)

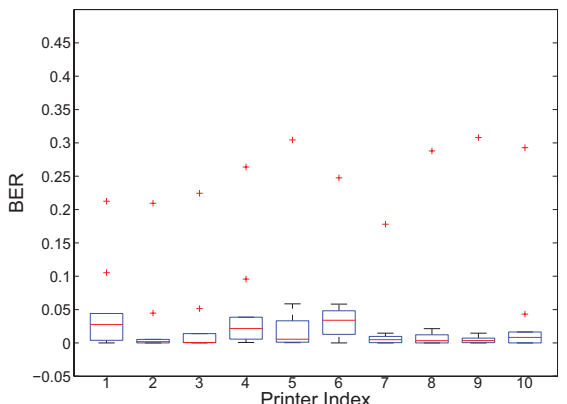

(e)

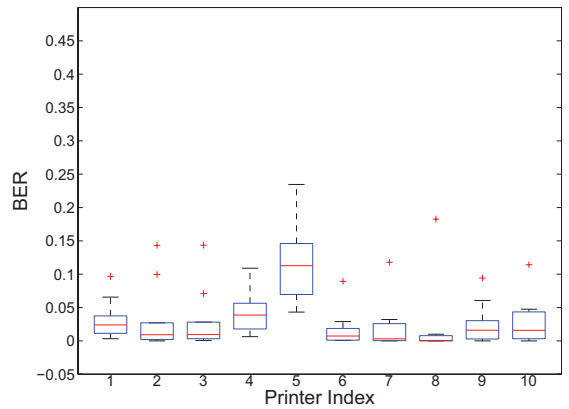

(h)

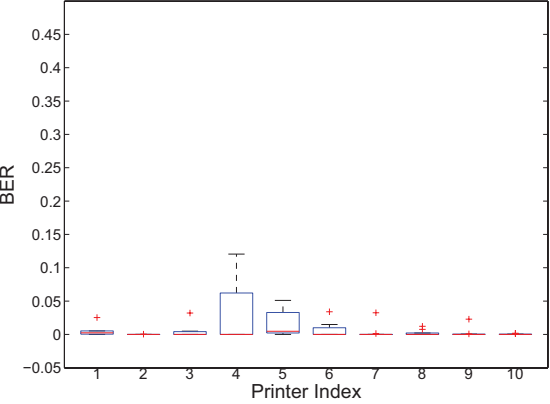

(c)

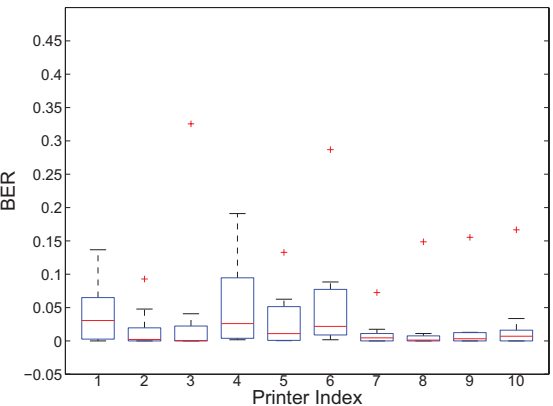

(f)

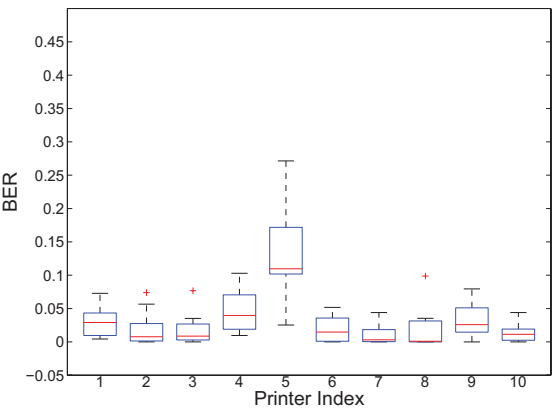

(i)

Fig. 8. Bit error rates for the proposed color QR codes across various printers and barcode sizes. (a) $20 \times 20 \mathrm{~mm}$, cyan. (b) $25 \times 25 \mathrm{~mm}$, cyan. (c) $30 \times 30 \mathrm{~mm}$, cyan. (d) $20 \times 20 \mathrm{~mm}$, magenta. (e) $25 \times 25 \mathrm{~mm}$, magenta. (f) $30 \times 30 \mathrm{~mm}$, magenta. (g) $20 \times 20 \mathrm{~mm}$, yellow. (h) $25 \times 25 \mathrm{~mm}$, yellow. (i) $30 \times 30 \mathrm{~mm}$, yellow.

The decoding and synchronization performance for Aztec codes are shown in Table V. The performance of the Aztec code is significantly impacted by the synchronization performance as part of the synchronization data (specifically size of the barcode) is encoded in the data field and required to be decoded first to estimate the full geometry of the barcode. If the size information is corrupted, then the barcode geometry cannot be determined and synchronization fails. However, once the synchronization is achieved the data is always decoded as shown in Table V. In this setting, synchronization rate is therefore, a more useful measure, indicative of the performance for Aztec codes. In our experiments, the highest rates are obtained for the interference cancellation mechanism with adaptive thresholding. These are comparable to the $68 \%$ synchronization rate of the same algorithm used on monochrome Aztec barcodes.

We also evaluate the bit error rate performance of the barcodes before the errors are corrected. Figure 10 shows the bit error rate (BER) performance across different decoding methodologies, where the interference cancellation based methods mitigate the effect of color interference and, in all cases, offer performance superior to simple thresholding algorithms. Under idealized conditions, these BERs can also be used to estimate the capacity, i.e. the maximum rate at which data can be reliably carried by the barcodes, for the different realizations of the proposed scheme, which can then be compared and contrasted with the monochrome barcode scenario. Specifically, assuming that post interference cancellation the three colorant channels are independent and can be represented by a binary symmetric channel (BSC) [39, pp. 186] model, for the color $\mathrm{QR}$ code using $\mathrm{PB}+\mathrm{AT}$ instantiation of the proposed framework, the estimated capacity is 2.63 bits per encoding module of the barcode which is 2.69 times the estimated capacity of 0.98 for the monochrome setting. Similarly for the, color Aztec code using the same PB+AT instantiation of the proposed framework, the estimated capacity is 2.69 bits per encoding module of the barcode which is 2.85 times the estimated capacity of 0.94 for the monochrome 
TABLE IV

Percent SSRs and DSRs (S ynchronization And Decoding Success Rates) For Color QR Codes Utilizing the Proposed Interference

Cancellation (Int. Cancellation) Approaches (PB+AT, PB+LT, EM) and Alternative Approaches (AT, LT). For Benchmarking

Purposes, the Corresponding Rates for Monochrome Barcodes Captured in Similar Settings Are Also Indicated

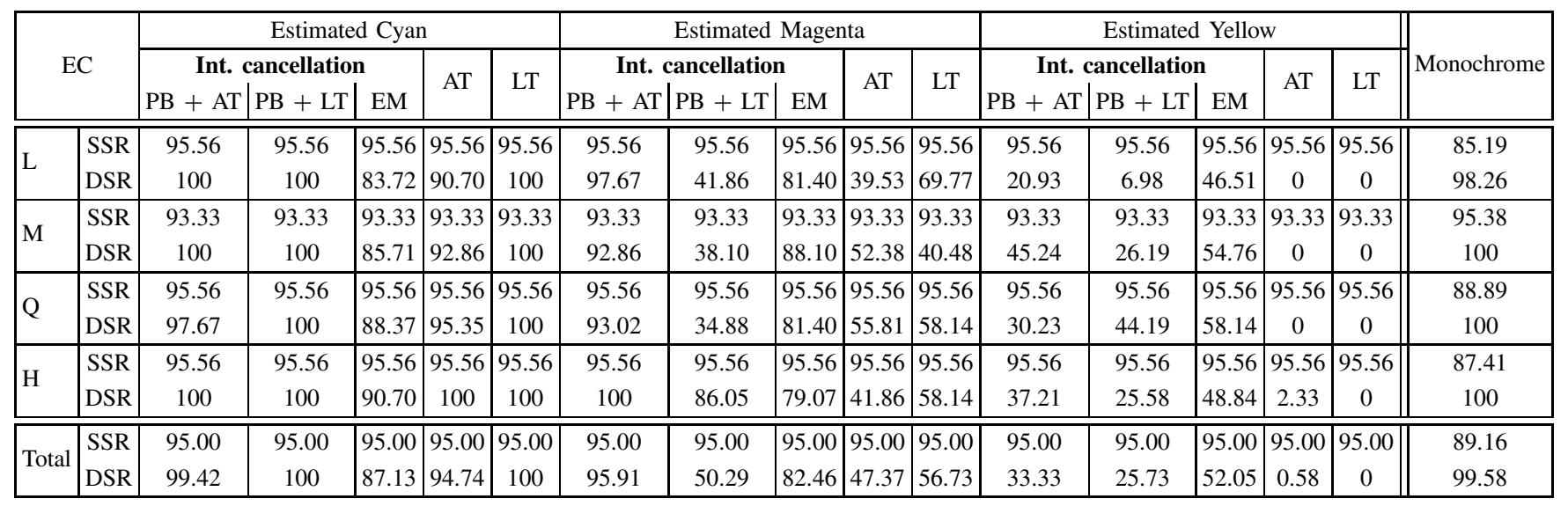

TABLE V

PERCENT SSRs and DSRs (Synchronization ANd DeCoding Success Rates) For Color AZTEC Codes Utilizing The Proposed interference Cancellation (Int. Cancellation) Approaches (PB+AT, PB+LT, EM) and Alternative Approaches (AT, LT). For Benchmarking Purposes, the Corresponding Rates for Monochrome Barcodes Captured in Similar Settings ARe Also Indicated

\begin{tabular}{|c|c|c|c|c|c|c|c|c|c|c|c|c|c|c|c|c|}
\hline & \multicolumn{5}{|c|}{ Estimated Cyan } & \multicolumn{5}{|c|}{ Estimated Magenta } & \multicolumn{5}{|c|}{ Estimated Yellow } & \multirow{3}{*}{ Monochrome } \\
\hline & \multicolumn{3}{|c|}{ Int. cancellation } & \multirow{2}{*}{ AT } & \multirow{2}{*}{ LT } & \multicolumn{3}{|c|}{ Int. cancellation } & \multirow{2}{*}{ AT } & \multirow{2}{*}{ LT } & \multicolumn{3}{|c|}{ Int. cancellation } & \multirow{2}{*}{ AT } & \multirow{2}{*}{ LT } & \\
\hline & $\mathrm{PB}+\mathrm{AT}$ & $\mathrm{PB}+\mathrm{LT}$ & EM & & & $\mathrm{PB}+\mathrm{AT}$ & $\mathrm{PB}+\mathrm{LT}$ & EM & & & $\mathrm{PB}+\mathrm{AT}$ & $\mathrm{PB}+\mathrm{LT}$ & EM & & & \\
\hline$\overline{\text { SSF }}$ & 75.56 & 77.78 & 55.26 & 75.56 & 75.56 & 75.56 & 55.56 & 55.26 & 77.78 & 48.89 & 57.78 & 33.33 & 42.11 & 4.44 & 0 & 67.91 \\
\hline SR & 100 & 100 & 100 & 100 & 100 & 100 & 100 & 100 & 100 & 100 & 100 & 100 & 100 & 0 & 0 & 100 \\
\hline
\end{tabular}

setting. Thus the three-fold increase in data rate for the proposed framework over the monochrome barcode setting also results results in a slightly less than three fold increase in the estimated capacity. Note that even though the EMtype algorithm achieves decoding and synchronization rates comparable with the pilot block approach, the bit error rate is significantly higher for the EM-type approach. The reason for the higher BERs, despite comparable decoding performance, is that the EM-type approach converges to a local optima while performing the interference cancellation, which can be quite different from the global optima and typically leads to a 50\% bit error rate in the cases where an incorrect local minimum is selected. In practical applications, the decoding success rate (DSR) is the more relevant metric and the EM algorithm offers performance close to the best with respect to this metric, while having the advantage of not requiring pilot blocks for parameter estimation.

\section{DISCUSSION}

The framework described in this paper can easily be adapted to most monochrome barcode schemes using at least one and potentially either of the proposed approaches for estimating the interference cancellation parameters. Many existing 2-D barcodes inherently have localization patterns to efficiently locate the barcode in the captured image. In current monochrome designs these patterns are uniformly colored, but they can be easily modified to carry the pilot blocks of known colorant combinations that are required to estimate the interference cancellation parameters. This dual use of localization patterns, to also carry interference cancellation pilot blocks, is natural for typical mobile application codes such as the QR or Aztec codes, where the size of the localization patterns is large, relative to the barcode area. Even in situations where the size of localization patterns is small, for example Data Matrix, it is still possible to embed and extract correct color estimates [40], [41]. For barcodes where this dual-use of the synchronization regions is either infeasible or undesirable, either our proposed EM-approach can be used to enable color interference cancellation without requiring the pilot blocks or, alternatively, part of the data field may be sacrificed and used to carry the pilot blocks. In this way, the data rate of any monochrome barcode can be tripled. In the appendix, we also evaluate the performance of the proposed EM-type color interference cancellation algorithm on the color barcodes recently proposed for high capacity barcode applications [10], where data is extracted from a barcode scanned on a flatbed scanner. As the results show in the appendix, the proposed EM-style interference cancellation algorithm significantly improves the BER performance of these color barcodes proposed for high capacity barcode applications, validating the generality of the framework proposed in this paper.

A color barcode in the proposed framework can be directly deployed with existing camera phones without requiring any hardware modifications because camera phones already 


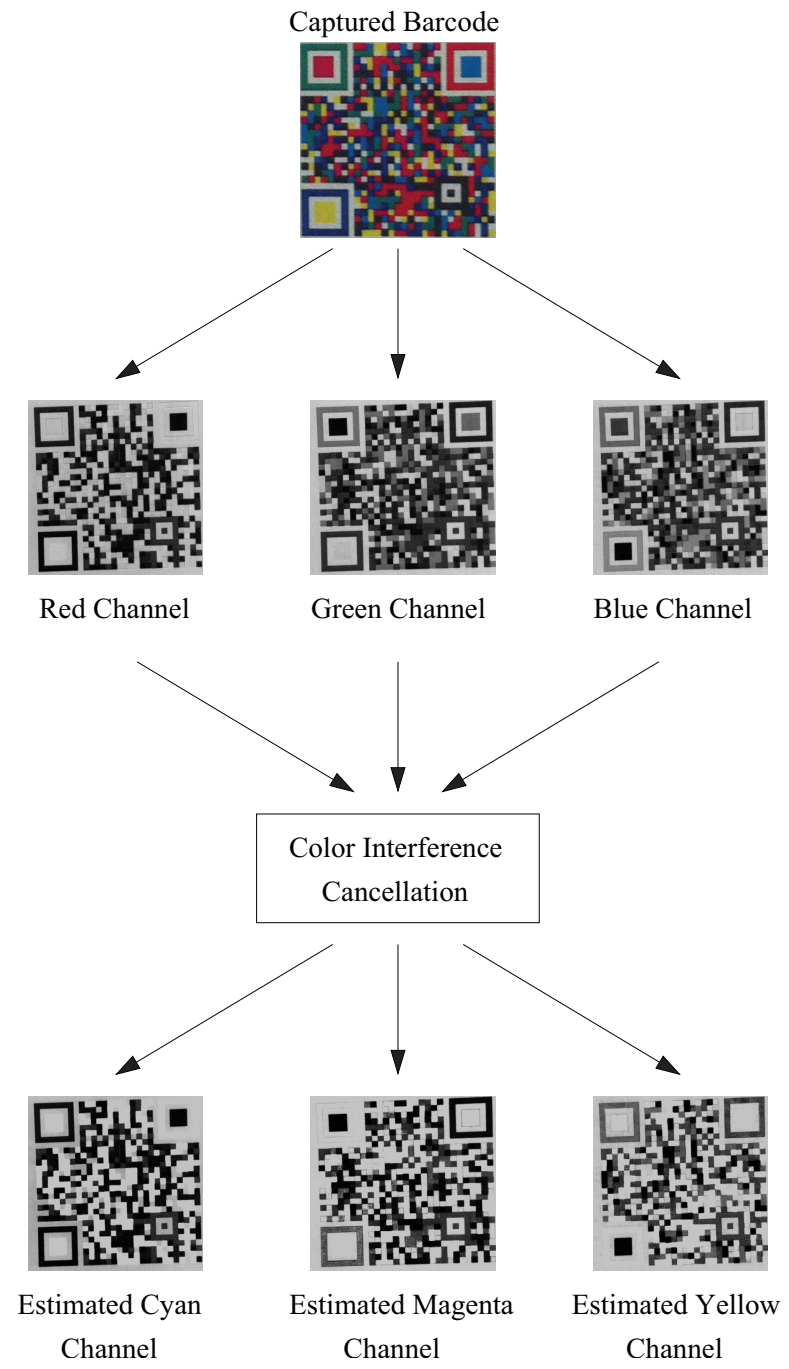

Fig. 9. Example color barcode capture and interference cancellation result.

capture color images with three R, G, B channels. On the decoder side, the migration to the new code can therefore be enabled simply by a software upgrade of existing barcode reading applications or by developing a new reader application. The software modification required is also minimal since the technique used for data embedding in each of the channels is the same as the method currently used with monochrome barcodes and only the front-end synchronization processing needs modification. On the encoder side, color printing functionality is required in order to enable the proposed color barcode, which cannot be reproduced with black and white printers. This is not a significant limitation for the proposed barcodes because color is already common in printing for the most common 2-D mobile barcode application scenarios.

The additional capacity provided by the proposed framework through embedding independent data in the print colorant channels is most effective for advertisement applications in the business world. In these applications, our construction of color barcodes can be used to embed primary advertisement data, such as a URL, in one colorant channel, and additional tagging data in the other colorant channels. This additional information can identify the advertisement channel and venue, without compromising the capacity, robustness, and ease of use. Upon decoding by a suitable application on the mobile phone, the color barcode can then, not only connect the user's mobile phone to the website with additional information, but can also communicate the tagging information to a server that gathers data on how many of the QR codes in each of the advertisement channels were actually captured by mobile phone users. This information can be subsequently mined to determine the effectiveness of different display and print advertising channels providing useful input for pricing, advertisement placement, etc.

In a number of applications, the three layers $(\mathrm{C}, \mathrm{M}$, and $\mathrm{Y}$ print layers) in the proposed construction of color barcodes can be used to advantageously encode three separate pieces of information. For instance, each of the $\mathrm{C}, \mathrm{M}$, and $\mathrm{Y}$ layers may encode a URL, providing three different URLs. A mobile phone reader application can then offer a user who captures the color barcode, the option to choose amongst these three URLs, thereby providing quick and ready access to three pieces of online information, without requiring the user to navigate or click on their small mobile device. For instance, the three simultaneous URLs may provide the user an option to connect to either an overall company website, to a website of the local store where the advertised merchandise is available, or to the website of the publication where the advertisement appears. The fact that the three layers can be independently generated in our proposed barcode framework, provides very significant design flexibility, which is also advantageous. Whereas the decoding process for the three colorant layers is loosely coupled, it does not pose limitations for their use.

The proposed solution does have a few limitations. Color barcodes designed in the proposed framework cannot be read by a monochrome barcode reader and the barcodes can therefore be used only with a suitably designed decoder. While, as demonstrated in the experiments, color barcodes are well protected against errors, if a barcode is damaged or stained, all three channels may be equally affected. Although we have not observed significant influence of the printer type on barcode decoding, in many home or office color printers the yellow channel contains a random dot pattern that is used for identifying the printer for anticounterfeiting purposes [38]. The presence of these random patterns explains lower performance of the framework in yellow channel. Also it makes our scheme more suitable for professional, industrial applications where much more control over color management is provided, as opposed to small print centers.

Note that although the proposed construction of color barcodes achieves reasonably high decoding rates (typically higher than $50 \%$ ), in some cases for a single image capture, they can not achieve the same decoding rate as the monochrome counterpart in all color channels. This however, is not a major problem because in many mobile applications a sequence of barcode images is captured, from which more than one frame can be used to extract the data in individual color channels.

In contrast with several other color barcode technologies that encode data in color, the proposed construction of color barcodes is significantly more robust to misregistration errors. 


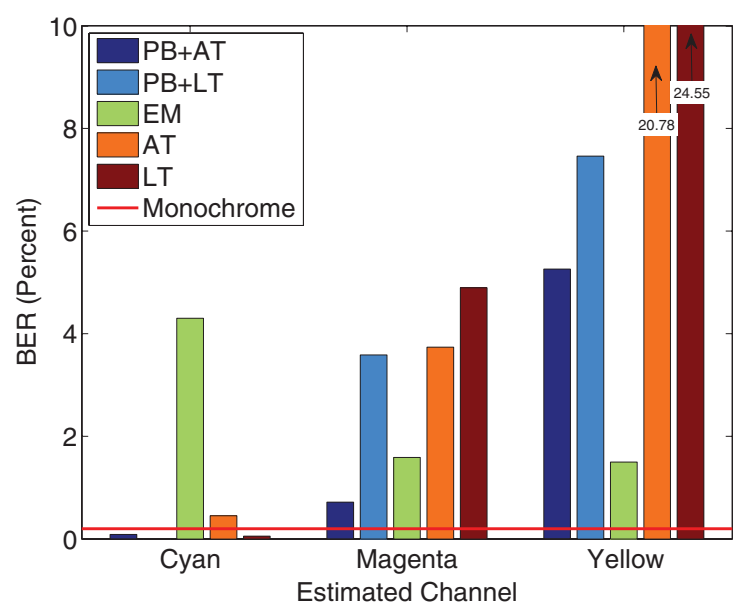

(a)



(b)

Fig. 10. BERs for color barcodes using the proposed interference cancellation approaches (PB+AT, PB+LT, EM) and alternative approaches (AT, LT). The BER for monochrome barcodes is also shown on the figure for comparison. (a) QR. (b) Aztec.

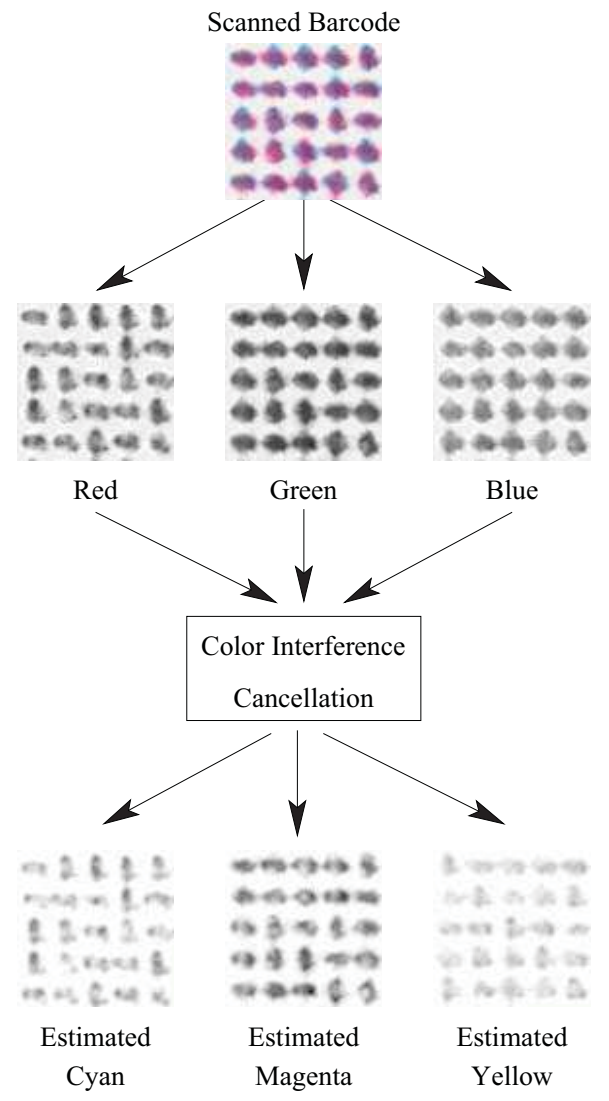

Fig. 11. Scanned RGB channels and estimated CMY channels after interference cancellation.

In the proposed construction of the color barcodes, independent data is encoded in print colorant layers and color interference cancellation is used at the decoder to overcome cross-channel coupling. This gives the method inherent robustness to mis-registration errors. While the layers may be misregistered, because the data is independently encoded and decoded from each layer it is robust against the misregistration, unlike other methods that rely on the color produced in combination of the overlay of the colorant layers (which changes with misregistration).

\section{CONClusion}

The framework proposed in this paper provides an effective method for extending monochrome barcodes to color. Our color code constructions offer three times the data rates of their monochrome counterparts, exploiting the spectral diversity provided by color printing and capture systems in conjunction with model-based interference cancellation that mitigates inter-channel coupling introduced by the physical characteristics of the devices. Although, bit error rates and therefore information capacities vary across the three resulting channels, the error rates are in ranges that are readily handled by the error correction coding options available for monochrome barcodes.

\section{APPENDIX}

The versatility of the framework proposed in this paper is further illustrated by the fact that the methodology can also be applied to high capacity color barcodes recently proposed in [10], an application that we report in related recent work [2]. The high capacity barcodes in [10] are intended for document workflow rather than mobile applications, where the data is to be recovered from a scanner rather than a digital camera capture of the barcode. Data is encoded in periodic elliptical dot arrays by modulating the orientation of the dots to encode data in C, M, and Y print-colorant channels. The overall color barcode is obtained when these color separations are printed in overlay. The spectral overlap between the print colorant and scanner sensor channels however, impacts the performance of these barcodes while extracting the data from R, G, and B scan channels. In this appendix, we show the benefit of the framework proposed in this paper to mitigate the impact of undesired color interference seen in the color channels of the high capacity barcodes proposed in [10].

For this purpose, a $2 \times 2$ inch color barcode was generated and printed on a HP Color 4700 printer with an array periodicity of 75 cells per inch (cpi) along horizontal and vertical directions for each of the CMY channels. This yields cell sizes (in printer pixel units) that provide reasonable flexibility to orient a dot along different directions within a cell and an 
TABLE VI

Bit Error Rates in Color Channels, Obtained From RGB SCAN Channels ANd By Utilizing The Proposed Color Interference CANCELlation Algorithm

\begin{tabular}{|c|c|c|c|}
\cline { 2 - 4 } \multicolumn{1}{c|}{} & $\begin{array}{c}\text { Estimated } \\
\text { Cyan }\end{array}$ & $\begin{array}{c}\text { Estimated } \\
\text { Magenta }\end{array}$ & $\begin{array}{c}\text { Estimated } \\
\text { Yellow }\end{array}$ \\
\hline $\begin{array}{c}\text { Without Int. } \\
\text { Cancellation }\end{array}$ & 0.10 & 0.03 & 0.28 \\
\hline $\begin{array}{c}\text { Proposed EM-style } \\
\text { Cancellation }\end{array}$ & 0.10 & 0 & 0.10 \\
\hline
\end{tabular}

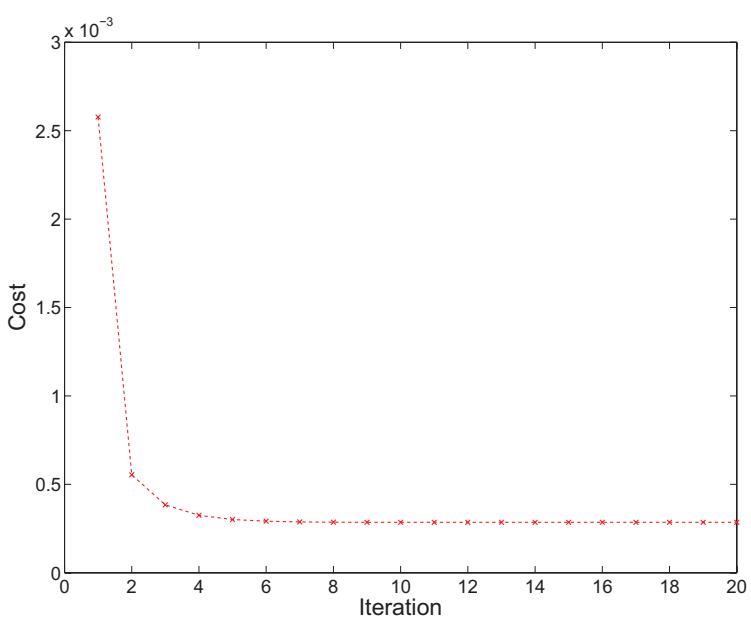

Fig. 12. Objective function versus iteration number illustrating convergence behavior of the proposed estimation algorithm.

overall data density of 16,875 bits per square inch. In each colorant channel we modulate the dot orientation along vertical or horizontal directions (i.e. $0 / 90^{\circ}$ orientations) based on the randomly generated data to be encoded in the barcode. The barcode was scanned on an HP Scanjet 8270 scanner with a scanning resolution of 1200 dots per inch (dpi) for data extraction $^{14}$. Figure 11 illustrates a portion of the scanned barcode and the color channels of the barcode before and after color interference cancellation algorithm. Note that the estimated cyan, magenta, and yellow channels with the proposed algorithm for color interference cancellation exhibit reduced color interference compared with the original RGB channels. The interference and the reduction are more pronounced when the dot orientations in the neighboring colorant channels in spectra (i.e. cyan-magenta or magenta-yellow), differ from each other.

Next we evaluate the bit error rates for the data embedded in each of the colorant channels in the barcodes, comparing the performance with and without color interference cancellation. The data is extracted from the estimated print colorant channels. When the proposed color interference cancellation is not employed, the data is extracted directly from $R, G$, and $\mathrm{B}$ channels of the scanned barcode complementary to the $\mathrm{C}, \mathrm{M}$, and Y print colorant channels, respectively. In each color channel, we employ Otsu's thresholding algorithm to mitigate the effect of color interference and reduce the print-scan noise

\footnotetext{
${ }^{14}$ Preliminary tests have also been conducted on other printer and scanner combinations with results similar to our chosen combination.
}

that impacts the estimated orientation of elliptical-dots. After global and local synchronization, the data is extracted from the thresholded color channels, utilizing image moments as detection statistics as detailed in [10]. Table VI lists the bit error rate performance in estimated CMY colorant channels with and without color interference cancellation. Note that the proposed algorithm for interference cancellation significantly improves the bit error rate performance especially in magenta and yellow channels, which are typically impacted more by the color interference [10]. Also note that the bit error rate performance in the cyan channel is not improved by the proposed algorithm as the red scan channel captures the cyan colorant with minimal interference from the other colorants [10], which is typically eliminated with the adaptive thresholding.

The convergence behavior of the algorithm is illustrated in Fig. 12, where the graph shows a plot of the optimization objective function as a function of the iteration number. Consistent with the remarks of Section II-B.2.b, monotone convergence to a (local) minimum (in the vicinity of the initialization) is observed.

\section{REFERENCES}

[1] O. Bulan, H. Blasinski, and G. Sharma, "Color QR codes: Increased capacity via per-channel data encoding and interference cancellation," in Proc. IS\&T/SID 19th Color Imag. Conf., Color Sci. Eng. Syst., Technol., Appl., Nov. 2011, pp. 156-159.

[2] O. Bulan and G. Sharma, "Improved color barcodes via expectation maximization style interference cancellation," in Proc. IEEE Intl. Conf. Acoust. Speech Signal Process., Mar. 2012, pp. 1509-1512.

[3] Information Technology-Automatic Identification and Data Capture Techniques-EAN/UPC Bar Code Symbology Specification. (2011, Jan.) [Online]. Available: http://www.iso.org/iso/isocatalogue/ catalogueics/cataloguedetailics.htm?csnumber $=46143$

[4] H. Kato and K. T. Tan, "Pervasive 2D barcodes for camera phone applications," IEEE Pervasive Comput., vol. 6, no. 4, pp. 76-85, Oct.-Dec. 2007.

[5] A. Madhavapeddy, D. Scott, R. Sharp, and E. Upton, "Using cameraphones to enhance human-computer interaction," in Proc. 6th Int. Conf. Ubiquitous Comput. 2004, pp. 1-2.

[6] M. Hara, M. Watabe, T. Nojiri, T. Nagaya, and Y. Uchiyama, "Optically readable two-dimensional code and method and aparatus using the same," U.S. Patent 5726435, Mar. 10, 1995.

[7] Information Technology-Automatic Identification and Data Capture Techniques-QR Code 2005 Bar Code Symbology Specification. (2011, Jan.) [Online]. Available: http://www.iso.org/iso/isocatalogue/ catalogueics/cataloguedetailics.htm?csnumber $=43655$

[8] D. Parikh and G. Jancke, "Localization and segmentation of a 2D high capacity color barcode," in Proc. IEEE Workshop Appl. Comput. Vis., Jan. 2008, pp. 1-6.

[9] J. Mayer, J. Bermudez, A. Legg, B. Uchoa-Filho, D. Mukherjee, A. Said, R. Samadani, and S. Simske, "Design of high capacity 3D print codes aiming for robustness to the PS channel and external distortions," in Proc. IEEE Int. Conf. Image Process., Nov. 2009, pp. 105-108.

[10] O. Bulan and G. Sharma, "High capacity color barcodes: Per channel data encoding via orientation modulation in elliptical dot arrays," IEEE Trans. Image Process., vol. 20, no. 5, pp. 1337-1350, May 2011.

[11] E. Schubert and A. Schroeder, "Vision-based reading system for colorcoded bar codes," Proc. SPIE, Mach. Vis. Appl. Ind. Inspect., vol. 2665, pp. 59-68, Jan. 1996.

[12] A. Grillo, A. Lentini, M. Querini, and G. F. Italiano, "High capacity colored two dimensional codes," in Proc. Int. Multiconf. Comput. Sci. Inf. Tech., Oct. 2010, pp. 709-716.

[13] S. Lyons and F. R. Kschischang, "Two-dimensional barcodes for mobile phones," in Proc. 25th Biennial Symp. Commun., May 2010 , pp. 344-347.

[14] S. J. Simske, J. S. Aronoff, and M. Sturgill, "Revenge of the physical - mobile color barcode solutions to security challenges," in Proc. Opt. Document Security, Jan. 2010, pp. 184-197. 
[15] H. Kato, K. T. Tan, and D. Chai, "Novel colour selection scheme for 2D barcode," in Proc. Int. Symp. Intell. Signal Comm. Syst., Jan. 2009, pp. 529-532.

[16] H. Kato, K. Tan, and D. Chai, Barcodes for Mobile Devices. Cambridge Univ. Press, New York: NY, 2010.

[17] S. J. Simske, J. S. Aronoff, M. M. Sturgill, and J. C. Villa, "Spectral pre-compensation and security deterrent authentication," in Proc. 24 Int. Conf. Digital Print. Technol., Sep. 2008, pp. 792-795.

[18] H. Bagherinia and R. Manduchi, "A theory of color barcodes," in Proc. IEEE Comput. Vis. Workshops, Nov. 2011, pp. 806-813.

[19] B. Oztan, G. Sharma, and R. P. Loce, "Misregistration sensitivity in clustered-dot color halftones," J. Electron. Imag., vol. 17, no. 2, pp. 023004-1-023004-30, Jun. 2008.

[20] K. Tan, D. Chai, H. Kato, and S. Ong, "A color 2D-barcode for mobile applications: Design tips," IEEE Pervasive Comput., vol. 11, no. 99, pp. 50-55, Feb. 2012

[21] K. Nurwono and R. Kosala, "Color quick response code for mobile content distribution," in Proc. 7th Int. Conf. Adv. Mob. Comput. Multimedia, Dec. 2009, pp. 267-271.

[22] S. Simske, M. Sturgill, and J. Aronoff, "Effect of copying and restoration on color barcode payload density," in Proc. 9th ACM Symp. Document Eng., 2009, pp. 127-130.

[23] T. Wakahara and N. Yamamoto, "Image processing of 2-dimensional barcode," in Proc. 14th Int. Conf. Netw.-Based Inf. Syst., Sep. 2011, pp. $484-490$.

[24] G. Sharma and H. J. Trussell, "Digital color imaging," IEEE Trans. Image Process., vol. 6, no. 7, pp. 901-932, Jul. 1997.

[25] "The 2D data matrix barcode," Comput. Control Eng. J., vol. 16, no. 6, p. 39, Jan. 2005.

[26] F. Grum and C. J. Bartleson, Optical Radiation Measurements: Color Measurement. New York: Academic, 1980.

[27] S. K. Ong, D. Chai, and K. T. Tan, "The use of border in colour 2D barcode," in Proc. Int. Symp. Parallel Distrib. Process. Appl., Dec. 2008, pp. 999-1005.

[28] J. A. C. Yule, Principles of Color Reproduction, Applied to Photomechanical Reproduction, Color Photography, and the Ink, Paper, and Other Related Industries. New York: Wiley, 1967.

[29] G. Sharma, "Set theoretic estimation for problems in subtractive color," Color Res. Appl., vol. 25, no. 4, pp. 333-348, Oct. 2000

[30] B. Oztan and G. Sharma, "Per-separation clustered-dot color halftone watermarks: Separation estimation based on spatial frequency content," J. Electron. Imag., vol. 19, no. 4, pp. 043007-1-043007-22, Dec. 2010.

[31] X. W. Chang and Q. Han, "Solving box-constrained integer least squares problems," IEEE Trans. Wireless Commun., vol. 7, no. 1, pp. 277-287, Jan. 2008.

[32] C. M. Bishop, Pattern Recognition and Machine Learning. New York: Springer-Verlag, 2006.

[33] Information Technology-Automatic Identification and Data Capture Techniques-Aztec Code Bar Code Symbology Specification. (2011, Apr.) [Online]. Available: http://www.iso.org/iso/isocatalogue/ cataloguetc/cataloguedetail.htm?\%csnumber $=41548$

[34] A. Longacre and R. Hussey, "Two dimensional data encoding structure and symbology for use with optical readers," U.S. Patent 5591956 , Jan. 7, 1997.

[35] S. Simske and J. Aronoff, "Qualification of a layered security print deterrent," J. Imag. Sci. Technol., vol. 51, no. 1, pp. 86-95, Feb. 2007.

[36] Y. Liu and M. Liu, "Automatic recognition algorithm of quick response code based on embedded system," in Proc. 6th Int. Conf. Intell. Syst. Design Appl., Oct. 2006, pp. 783-788.

[37] Y. Liu, J. Yang, and M. Liu, "Recognition of QR code with mobile phones," in Proc. Chin. Control Decis. Conf., Jul. 2008, pp. 203-206.

[38] Machine Identification Code Technology in Color Laser Printers. (2009, Dec.) [Online]. Available: http://www.eff.org/wp/investigating-machineidentification-code-technology-color-laser-printers.html

[39] T. Cover and J. A. Thomas, Elements of Information Theory. 2nd ed. New York: Wiley, 2006

[40] W. Claycomb and D. Shin, "Using a two dimensional colorized barcode solution for authentication in pervasive computing," in Proc. ACS/IEEE Int. Conf. Pervasive Services, Jun. 2006, pp. 173-180.

[41] K. Tan, D. Chai, H. Kato, and S. Ong, "Designing a color barcode for mobile applications," IEEE Pervasive Comput., vol. 11, no. 2, pp. 50-55, Feb. 2012.

[42] S. J. Simske, J. S. Aronoff, and M. Sturgill, "Revenge of the physicalmobile color barcode solutions to security challenges," Hewlett-Packard Laboratory, Palo Alto, CA, Tech. Rep. HPL-2010-7, Jan. 2010.

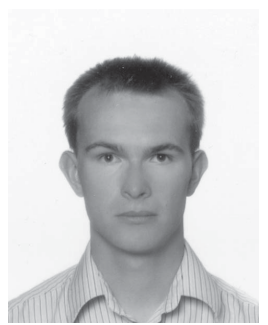

Henryk Blasinski (S'10) received the M.S. degree (Hons.) in telecommunications and computer science from the Lodz University of Technology, Lodz, Poland, and the Diplome d'Ingeneiur degree from the Institut Superieur d'Electronique de Paris, Paris, France, in 2008 and 2009, respectively. He is currently pursuing the Ph.D. degree with the Department of Electrical Engineering, Stanford University, Stanford, CA

He was a Fulbright Scholar with the Department of Electrical and Computer Engineering, University of Rochester, Rochester, NY, from 2010 to 2011. His current research interests include image processing as well as human and computer vision.

Mr. Blasinski is a recipient of several fellowships and awards, including the Fulbright Fellowship, the Fellowship from the Minister of Higher Education of the Republic of Poland, the Polish Talents Award, the DP Systems Award, the Fellowship of the Lodz Region Marshall, and the Crawford Prize for the best M.S. project.

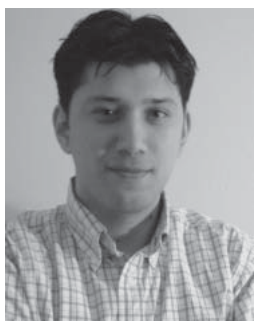

Orhan Bulan received the B.S. degree (Hons.) in electrical and electronics engineering from Bilkent University, Ankara, Turkey, in 2006, and the M.S. and Ph.D. degrees in electrical and computer engineering from the University of Rochester, Rochester, NY in 2007 and 2011, respectively.

He is currently a Post-Doctoral Research Associate with Xerox Research Center, Webster, NY, where he was also a Research Intern in the summers of 2009, 2010, and 2011. His current research interests include computer vision, machine learning, signal and image processing, and multimedia security.

Dr. Bulan is a recipient of the Best Student Paper Award at the 2008 Western New York Image Processing Workshop organized by the Rochester Chapter of the IEEE Signal Processing Society.

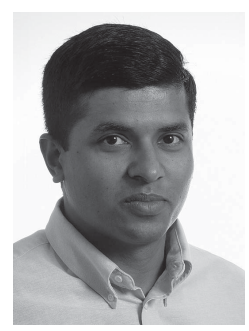

Gaurav Sharma (S'88-M'96-SM'00-F'13) received the B.E. degree in electronics and communication engineering from the Indian Institute of Technology Roorkee (formerly the University of Roorkee), Roorkee, India, and the M.E. degree in electrical communication engineering from the Indian Institute of Science, Bangalore, India, in 1990 and 1992, respectively, and the M.S. degree in applied mathematics and the Ph.D. degree in electrical and computer engineering from North Carolina State University, Raleigh, in 1995 and 1996, respectively.

$\mathrm{He}$ is currently an Associate Professor with the University of Rochester, Rochester, NY, where he is with the Department of Electrical and Computer Engineering, the Department of Biostatistics and Computational Biology, and the Department of Oncology, and he was the Director for the Center for Emerging and Innovative Sciences, a New York state funded center for promoting joint university-industry research and technology development, from 2008 to 2010. From 1996 to 2003, he was with Xerox Research and Technology, Webster, NY, as a Member of Research Staff and then a Principal Scientist. He is the Editor of the Color Imaging Handbook (CRC press, 2003). His current research interests include media security and watermarking, color science and imaging, genomic signal processing, and image processing for visual sensor networks.

$\mathrm{He}$ is a fellow of SPIE, a member of Sigma Xi, Phi Kappa Phi, Pi Mu Epsilon, the IS\&T, and the Signal Processing And Communications Societies of the IEEE. He was the Technical Program Chair for the 2012 IEEE International Conference on Image Processing, and the 2010-2011 Chair of the IEEE Signal Processing Society's Image Video and Multi-dimensional Signal Processing Technical Committee, the 2007 Chair of the Rochester Section of the IEEE and the 2003 Chair of the Rochester Chapter of the IEEE Signal Processing Society. He is a member on the IEEE Signal Processing Society's Information Forensics and Security Technical Committee and an Advisory Member on the IEEE Standing Committee of Industry DSP. He is the Editor-in-Chief of the Journal of Electronic Imaging and was an Associate Editor of the Journal of Electronic Imaging, the IEEE TRANSACTIONS ON IMAgE PROCESSING, and the IEEE TRANSACTIONS ON INFORMATION FORENSICS AND SECURITY. 